\title{
Bacillithiol disulfide reductase Bdr - insight into a new type of FAD-containing NADPH- dependent oxidoreductases
}

\author{
M. Hammerstad ${ }^{1}$, I. Gudim ${ }^{1}$, H.-P. Hersleth ${ }^{1,2}$ \\ ${ }^{1}$ University of Oslo, Department of Biosciences, Oslo, Norway, \\ ${ }^{2}$ University of Oslo, Department of Chemistry, Oslo, Norway
}

h.p.hersleth@ibv.uio.no

Low G+C Gram-positive Firmicutes, such as the clinically important pathogens Staphylococcus aureus and Bacillus cereus, use the low-molecular weight (LMW) thiol bacillithiol (BSH) as a defense mechanism to buffer the intracellular redox environment and counteract oxidative stress encountered by human neutrophils during infections. The protein bacillithiol disulfide reductase Bdr has recently been shown to function as an essential NADPH-dependent reductase of oxidized bacillithiol disulfide (BSSB) resulting from stress responses and is crucial in maintaining the reduced pool of BSH and cellular redox balance. We have solved the first structures of Bdrs, namely from $S$. aureus and $B$. cereus [1]. Our analyses reveal a uniquely organized biological tetramer; however, the monomeric subunit has high structural similarity to other flavoprotein disulfide reductases. The absence of a redox active cysteine in the vicinity of the FAD isoalloxazine ring implies a new direct disulfide reduction mechanism, which is backed by the presence of a potentially gated channel, serving as a putative binding site for BSSB in proximity to the FAD cofactor. We also report enzymatic activity for both Bdrs, which along with the structures presented in this work provide important structural and functional insight into a new class of FAD-containing NADPH-dependent oxidoreductases, related to the emerging fight against pathogenic bacteria.

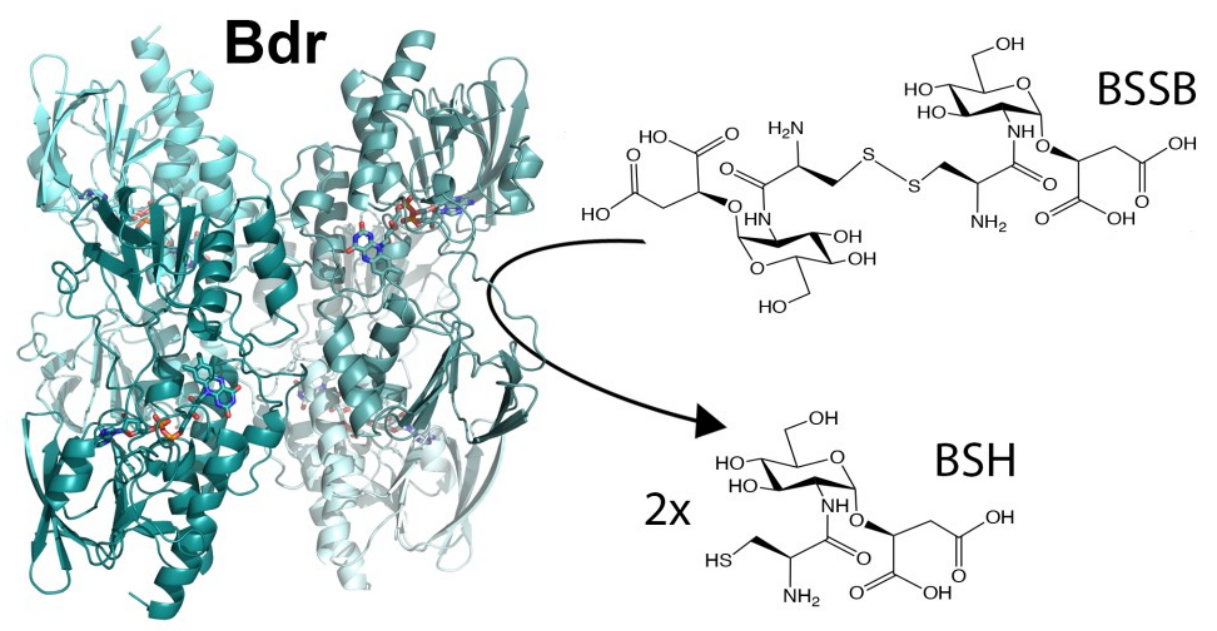

Figure 1. Reduction of BSSB by Bdr

[1] Hammerstad, M., Gudim, I. \& Hersleth, H.-P. (2020). Biochemistry. 59, 4793-4798.

Keywords: oxidoreductase; bacillithiol; flavoprotein; structure; enzymology 